\title{
Eigenartige längere Zeit an kleiner Hautstelle lokalisierte familiäre Psoriasis nebst Bemerkungen über die Ätiologie dieser Krankheit.
}

\author{
Von \\ Erich Hoffmann.
}

(Eingegangen am 11. Februar 1921.)

Herxheimer1) hat auf Grund seiner großen Erfahrung öfters darauf hingewiesen, daß Hautflechten wie Lichen ruber zunächst an bestimmten Stellen wie den Genitalien lokalisiert auftreten können, um sich erst nach einiger Zeit allgemein auszubreiten. Dies Verhalten hat er mit dem der syphilitisehen Infektion verglichen und gemeint, daß es für die Auffassung des Lichen ruber als parasitärer Erkrankung spräche. Wenn er angibt, daß nun noch der Beweis der Ansteckung bei primär genitalem Auftreten des lichen ruber zu erbringen sei, so kann ich auf Grund meiner Beobachtungen sagen, daß trotz langen Bestehens eines Lichen planus an den Genitalien oder der Mundschleimhaut und reichlichster Gelegenheit zur Übertragung, z. B. zwischen Eheleuten, ich nie eine solche habe feststellen können. Ohne aber auf diese Frage und die einschlägige Literatur eingehen zu wollen, möchte ich hier über Fälle von Psoria sis berichten, die in mancher Hinsicht Interesse verdienen und ebenfalls längere Zeit auf eine bestimmte umschriebene Örtlichkeit beschränkt blieben.

Der erste Fall betrifft eine 63 jährige Arztfrau, die von frühester Jugend an spröder Haut und Seborrhoea capitis litt. Dezember 1907 entstand eine Erkrankung um den Nagel der rechten großen Zehe, die sich alsbald zu einer scheibenförmig begrenzten schuppenden, bisweilen ein wenig nässenden Fläche ausdehnte, und der nach gut einem Jahr eine ähnliche Entzündung am linken Ringfinger folgte. Außerdem wies die Pat. Gichtknoten an den Fingergelenken auf und war hereditär stark belastet, da ihre Mutter an schwerer Gicht und später auch an einer schuppenden Flechte am ganzen Körper Iange Zeit gelitten hat. Im Juni 1911 sah ich die Kranke zum erstenmal. Wegen des hart. näckigen, allen Mitteln trotzenden Ausschlages an der Zehe, der allmählich zum. Verlust des Nagels geführt hatte, und wegen der scharfen Begrenzung war die Erkrankung für ein krebsartiges Leiden, und zwar als Pagetsche Erkrankung angesehen worden. Ein hervorragender Dermatologe und sehr bekannter Chirurg hatten deshalb die Amputation der Zehe als notwendig angeraten. Damals

1) Archiv für Dermatol. u. Syphilis 11 (Festschrift für Edmund Lesser), S. 411 . 
fand sich eine scharf u mschriebene, bogig begrenzte, wenig infiltrierte, bläulichrote, flächenhafte Veränderung, die die Dorsalseite der rechten großen Zehe ganz, die Plantarseite etwa zur Hülfte einnahm und mit Schuppen und Schuppenkrusten bedeckt war. Der Nagel fehlte und war durch graugrünliche Schuppenmassen ersetzt. Am linken Ringfinger bestand eine ähnliche Erkrankung des Endgliedes, während der Nagel nur von gelblich und graugrünlich gefärbten Herden durchsetzt war.

Ich konstatierte damals Gicht und eine atypische Psoriasis und riet deshaIb von der Operation dringend ab. Die Pat. wurde nun eine Reihe von Wochen in der Klinik behandelt und eine erhebliche Besserung mit antipsoriatischen Mitteln erzielt, wenn auch die völlige Beseitigung nicht gelang. Nach wechselnden Rückfällen der örtlichen Erkrankung trat dann im Januar 1913 eine allge mei ne Eruption von unzweifelhaft psoriatischem Charakter, aber mit Neigung zu Schuppenkrustenbildung ein, die sich in den folgenden Jahren noch mehrfach wiederholte. Durch Chrysarobin, Pyrogallol, Hydrarg. praec. alb. und Röntgen. bestrahlung gelang es immer wieder, die Erkrankung im Zaum zu halten. Während des Krieges erfolgte ein bedeutender Rückgang und die Pat. blieb bis zu ihrem im September 1919 an Carcinoma mammae erfolgten Tode fast frei von Erscheinungen. Selbst die Nagelerkrankung besserte sich sehr.

Der zweite Fall betrifft den Sohn der Kranken, einen Chirurgen. Auch er litt von seiner Kindheit an an Seborrhoea capitis und in den Pubertätsjahren an einem seborrhoischen Ekzem der Nasenfurche, das 1899 sich weiter über den Körper ausbreitete und von Doutrelepont als seborrhoisches Ekzem diagnostiziert und behandelt wurde. Auch später blieb Neigung zu Schuppung bisweilen auch im Gesicht bestehen. Im Juli 1912 sah ich den Kranken zuerst und stellte eine psoriatische Erkrankung mehrerer Fingernägel neben einer nur wenig typischen Psoriasis ca pitis fest. Im Juli 1913 war die Erkrankung völlig unverkennbar und immer noch lediglich a n den Fingernägeln und einzelnen Stellen der Kopfhaut lokalisiert. Röntgenbestrahlungen und Salben besserten das Leiden, aber beseitigten es nicht ganz. Im Krieg trat dann auch bei ihm eine weitgehende Besserung und fast völliges Schwinden der Psoriasis ein und erst gegen Schluß des Krieges zeigte sich wieder ein Herd am Gesäß als Folge anhaltenden Reitens. Im Oktober 1920 fand ich wiederum eine Psoriasis aller Fingernägel und des Kopfes und daneben typische Herde am GesäB, den Ellenbeugen, am Penis und Scrotum.

Ein anderer im Krieg gefallener Bruder, den ich selbst nicht untersucht habe, litt ebenfalls an hartnäckiger Seborrhoe der Kopfhaut; eìne Psoriasis soll bei ihm nicht bestanden haben. Eine Schwester ist ebenfalls von Psoriasis stets frei geblieben und hat nur vorübergehend einmal ein Ekzem an Händen und Armen nach starkem Waschen gehabt, das aber bald abheilte. 2 Kusinen mütterlïcherseits sollen ebenfalls zu $\mathrm{Ekzem}$ an den Händen neigen.

Wir haben somit eine familiäre Erkrankung an Psoriasis vor uns, die bei der ersten Patientin über ein Jahr lang nur auf die große Zehe lokalisiert blieb und eine Pagetsche Erkrankung: vortäuschte, dann noch mehrere Jahre auf die genannte Zehe und ein Fingernagelglied beschränkt blieb. Bei dem Sohn der Patientin war die Erkrankung ebenfalls lange Zeit nur auf einige Stellen des Kopfes und die Fingernägel lokalisiert. Bemerkenswert bei der ersten Patientin ist die Kombination mit langdauernder und hartnäckiger Gicht und die erblic he Belastung mit Gicht und Schuppen- 
flechte. Eine solche streng auf kleine Stellen des Körpers für lange Zeit lokalisierte Psoriasis ist an anderen Orten der Haut keine Seltenheit, Vielmehr ergibt die Untersuchung psoriatischer Familien öfters, daß einige Mitglieder viele Jahre lang oder ihr ganzes Leben hindurch nur einen Herd an Ellenbogen oder Knien zeigen. Aber auch an anderen Stellen, z. B. den Fingernägeln, Handflächen ${ }^{1}$ ), dem Kopf und den Genitalien kommt eine solche lokalisierte Psoriasis vor ${ }^{2}$ ). Abgesehen von den diagnostischen Schwierigkeiten ${ }^{3}$ ), die solche Fälle bereiten, sind sie auch für die Pathogenese der Psoriasis von Interesse. Allerdings darf man meiner Meinung nach daraus nicht ohne weiteres schließen, daß die Psoriasis eine parasitäre Erkrankung ist, und daß von einem solchen primären Herd eine allgemeine Infektion des Körpers erfolgt. Diese Fälle sind vielmehr auch mit der Annahme einer St off wechselerkra $\mathbf{k} \mathbf{k} \mathbf{n g}$ vereinbar.

Ưber die Ätiologie der Psoriasis gehen die Meinungen ja noch immer weit auseinander. Die parasitäre Theorie in der Form, daß die Psoriasis eine den Dermatomykosen vergleichbare parasitäre und reine Hautkrankheit sei, hat allerdings viele Anhänger verloren. Ebenso wird die neuropathische Theorie wohl nur noch von wenigen anerkannt. Die Meinung neuerer Forscher geht dahin, daß die Psoriasis eine allge meine Erkrankung ist mit einer eigenartigen Beschaffenheit der Haut, die sie auf verschiedene Reize leicht mit Parakeratose reagieren läßt und deshalb als para keratotische Diathese bezeichnet wird. Die weitergehende Annahme Sambergers ${ }^{4}$, daß die andersartige Reaktion der Haut durch die gewöhnlichen Eiterkokken zustande komme, kann ich nicht teilen, da nach Untersuchungen an meiner Klinil frische Psoriasisefflorescenzen sich meist als steril erweisen und bei Übertragung auf Gesunde keine Pyodermie hervorzurufen pflegen. Auch die Experimente Lennhoffs ${ }^{5}$, wonach Einimpfung von Schuppenmaterial bei Psoriatikern leichter eine Impfpsoriasis erzeugt als ein-

1) Isolierte palmare Psoriasis von langjähriger Dauer habe ich mehrfach beobachtet; sie wurde meist verkannt.

2) Eine eingehendere Darstellung solcher Fälle gibt die Dissertation von Karl Adrian (Bonn 1921) aus meiner Klinik.

3) Auch umgekehrt wie in meinem Fall kann ein diagnostischer Irrtum vorkommen. So fand ich bei Übernahme der Bonner Klinik einen Patienten, der eine talergroße, umschriebene Erkrankung der Kreuzgegend seit mehreren Jahren aufwies, die von Doutrelepont als Psoriasis atypica diagnostiziert und behandelt worden war. Mir fiel sogleich die eigentümliche Form und geringe Schuppung sowie die Beschaffenheit des Randsaumes auf; die histologische Untersuchung zeigte dann auch, daß es sich um ein E pithelioma planum handelte.

$\left.{ }^{4}\right)$ Dermatol. Wochenschr. 1918, Nr. 41 u. 42.

5) Berl. blin. Wochenschr. 1920, Nr. 33. 
familiäre Psoriasis nebst Bemerlxungen über die Ätiologie dieser Krankheit. 231

faches Ritzen der Haut, sind nicht in dem Sinne eindeutig, wie er annimmt; denn auch chemische Stoffe könnten diese leichtere Reaktion bewirken. Besonderes Interesse verdienen weiter die Arbeiten von $\mathrm{Li}$ pschüt $\mathrm{z}^{1}$ ) und $\mathrm{Brock}^{2}$ ), von denen der erste ein der motropes Virus ähnlich dem der Geflügelpocke, der Vaccine usw. annimmt, während der zweite angibt, daß er durch Röntgenbestrahlung der Thymus mit einer Reizdosis einen Rückgang der Psoriasis erzielt habe, was ihn veranlaßt, eine endokrine Störung als Ưrsache der Psoriasis in Betracht zu ziehen. Die Frage spitzt sich nunmehr also dahin zu, ob ein dermotropes Virus oder ein dermotropes Stoffwechselprodukt für die Entstehung der Psoriasis verantwortlich zu machen ist. Ich selbst ${ }^{3}$ ) habe mich vor kurzem für die Auffassung der Psoriasis in letzterem Sinne ausgesprochen und die Psoriasis den Exkretionsder matosen, zu denen man schwere Arzneiexantheme ${ }^{4}$ ), diathetische Ekzeme usw. rechnen kann, nahegestellt. Sollte die Behaupturig Brocks, die wir allerdings nicht bestätigen können, sich bewahrheiten, so würde dies meiner Meinung nach eher gegen. als fürdie parasitäre Theorie der Psoriasis sprechen; denn die Konfusion dieser Behauptung mit der Sa mberger schen Hypothese möchte ich auf Grund meiner obigen Bemerkungen ablehnen. Der Satz, „Was Psoriasis ist, weiß kein Mensch", besteht also wohl noch zu Recht, aber vielleicht dürfen wir heute sagen: Entweder ein dermotropes Virus oder ein dermotropes Stoffwechselprodukt, das zuweilen auch arthrotrop ist ${ }^{5}$ ), dürfte für die Ätiologie der Psoriasis in Betracht kommen. ${ }^{6}$ )

1) Arch. f. Dermatol. u. Syphilis. 12\%, H. 4.

2) Strahlentherapie 11, H. 2.

3) Dermatol. Zeitschr. 31, Nr. 3, S. 143.

4) z. B. stark schuppende und nässende $S$ alvars a nexantheme.

5) Während des Krieges haben eine Anzahl meiner Patienten wohl infolge der andersartigen Ernährung einen Rückgang ihrer Schuppenflechte beobachtet; dies kann ebenso wie die Erfahrung, daß Psoriatiker, ähnlich wie asthmatische Ekzematöse, sich bei bestehendem Ausschlag wohler fühlen, im Sinne einer Stoffwechselerkrankung am leichtesten gedeutet werden.

$\left.{ }^{6}\right)$ Anm. bei der Korrektur: Ich verweise hier auf einen neverdings beobachteten Fall von bandförmiger Psariasis am Arm übereiner pyogenen Lymphangitis, den ich in der Niederrhein. Ges. f. Natur- $u$. Heilkunde am 9. 5. 21 vorgestellt habe (vergl. Protokoll D. M. W. 1921); die schnelle Entstehung der lichenoiden Psoriasis genau im Verlauf des Lymphstrangs war hier ebenso bemerkenswert als der schnelle Rückgang. 